\title{
Latent TGF-beta binding protein-1 plays an important role in craniofacial development
}

\section{Abstract}

Yiting XIONG"\# iD

Rongrong SUN ${ }^{1 \#}$ i Jingyu $\mathrm{LI}^{2}$ iD

Yue $\mathrm{WU}^{2}$

Jingju ZHANG ${ }^{1}$
Submitted: April 24, 2020 Modification: June 31, 2020

Accepted: July 29, 2020
Objective: This study aims to replicate the phenotype of $L t b p 1$ knockout mice in zebrafish, and to address the function of $L T B P 1$ in craniofacial development. Methods: Whole mount in situ hybridization (WISH) of Itbp1 was performed at critical periods of zebrafish craniofacial development to explore the spatial-temporal expression pattern. Furthermore, we generated morpholino based knockdown model of Itbp1 to study the craniofacial phenotype. Results: WISH of Itbp1 was mainly detected in the mandibular jaw region, brain trunk, and internal organs such as pancreas and gallbladder. And Itbp1 colocalized with both sox9a and ckma in mandibular region. Morpholino based knockdown of Itbp1 results in severe jaw malformation. Alcian blue staining revealed severe deformity of Meckel's cartilage along with the absence of ceratobranchial. Three-dimension measurements of Itbp1 morphants jaws showed decrease in both mandible length and width and increase in open mouth distance. Expression of cartilage marker sox9a and muscle marker ckma was decreased in Itbp1 morphants. Conclusions: Our experiments found that Itbp1 was expressed in zebrafish mandibular jaw cartilages and the surrounding muscles. The Itbp 1 knockdown zebrafish exhibited phenotypes consistent with Ltbp1 knockout mice. And loss of Itbp1 function lead to significant mandibular jaw defects and affect both jaw cartilages and surrounding muscles.

Keywords: $\angle T B P 1$. Craniofacial anomalies. Developmental biology. Zebrafish. 


\section{Introduction}

The latent transforming growth factor- $\beta$ binding protein-1 $(L T B P-1)$ is an extracellular matrix protein that is structurally similar to the fibrillin family. There are several human and mouse $L t b p 1$ splice variants including a short ( $L t b p-1 S)$ and long $(L t b p-1 L)$ form, which may arise from the two separated promoters, alternative RNA splicing, and differential proteolytic processing. ${ }^{1}$ LTBP1 is widely expressed among species in variable amounts in different tissues, including heart, lung, liver, placenta, skeletal muscle, and bone..$^{2,3}$ Current researches suggest that $\angle T B P 1$ is a multifunctional protein that bind latent transforming growth factor- $\beta$ (TGF- $\beta$ ) and regulate its function in bone and other connective tissues. ${ }^{4-10} \angle T B P 1$ is reported to ease the secretion of latent TGF- $\beta$ and modulate the activation of latent TGF- $\beta .{ }^{11-16}$ It is considered as a stable component of extracellular fibrillar structure that play a critical role in the storage and release of TGF- $\beta$ as a large latent TGF- $\beta$ complex. ${ }^{17-19}$ In addition to regulating the function of TGF- $\beta, \angle T B P 1$ function as a structural component of connective tissue microfibrils. ${ }^{20}$ Studies focusing on its function as matrix proteins find that $\angle T B P 1$ colocalize with fibrillin 1 in microfibrillar structures not only in the extracellular matrix of primary osteoblasts but also at the surface of newly forming osteoid and bone, suggesting that $L T B P 1$ play an important role in the formation of bone and connective tissue. ${ }^{8}$

The important function of $L T B P 1$ has been investigated by a series of studies. Targeted deletion of exon 1 or 2 led to $L t b p-1 L$-null mice dying shortly after birth from defects in heart organogenesis. ${ }^{21}$ These severe cardiac deformities include persistent truncus arteriosus and interrupted aortic arch, which are associated with imperfect function of cardiac neural crest cells. Similarly, targeted deletion of exon 8 to generate $L t b p 1 L$ knockout mice died perinatally, these mice also present heart defects including persistent truncus arteriosus and interrupted aortic arch which are similar to $L t b p 1 L$-null mice. ${ }^{22}$ These studies indicate the crucial role of $\angle T B P 1$ in the development of the cardiovascular system. Another Ltbp1 knockout mice is manipulated by targeted deletion of exon 5 , which is the first exon shared by the $L t b p 1 L$ and $L t b p 1 S .{ }^{23}$ These total knockout mice are viable and fertile, they exhibit craniofacial abnormalities consisting of more compact head structure with shorten maxilla and mandible. Furthermore, the total $L t b p 1$ knockout mice have shortened long bones. Moreover, LTBP1 knockout mice are less prone to hepatic fibrogenesis. ${ }^{23}$ These phenomena suggest that loss of $\angle T B P 1$ function lead to potential changes of the biological activity of TGF- $\beta$ in fibrogenesis action. Researchers also used the deletion of $L t b p 1 L$ exon 5 knockout model to address the function of Ltbp1 in female fertility. ${ }^{24}$ Since $L t b p 1$ knockout female mice showed impaired reproduction with subfertility and ovarian cyst formation, the interruption of TGF- $\beta$ function which would lead to defective follicular wound healing was suggested as the potential cause.

It is known that $\angle T B P 1$ is critically required for the cardiovascular system organogenesis, bone and connective tissue formation, female reproduction, and craniofacial development. ${ }^{21-24}$ However, the discrepancy of phenotypes in Ltbp 1 gene-manipulated mice is difficult to explain. Moreover, the function of $\angle T B P 1$ in craniofacial development remains unclear. Since the gene function, fundamental signaling pathways and cellular events in craniofacial morphogenesis have proven to be highly conserved among vertebrates from zebrafish and mouse to man, ${ }^{25,26}$ we chose zebrafish to observe the phenotype in another model and to address the function of $\angle T B P 1$ in craniofacial aspect. We performed whole mount in situ hybridization (WISH) of Itbp 1 in critical periods of zebrafish craniofacial development to explore the spatial-temporal expression pattern. Furthermore, we generated morpholino based knockdown model of Itbp 1 to study the craniofacial phenotype.

This study aims to replicate the phenotype of $L t b p 1$ knockout mice in zebrafish, and to address the function of $\angle T B P 1$ in craniofacial development.

\section{Methodology}

\section{Zebrafish husbandry}

Wild-type zebrafish (Tuebingen) were maintained with standard techniques. ${ }^{27}$ All experiments were performed in compliance with the Animal Ethics Committee.

Zebrafish embryos were collected from natural mating and kept at $28.5^{\circ} \mathrm{C}$. About 300 male/female zebrafish embryos were used for detection of Itbp 1 expression pattern at five experimental periods: 1 cell, sphere, shield, 1 day postfertilization (DPF), and 3 DPF 
stages. About 1800 male/female zebrafish embryos were used for Itbp1 knock-down phenotype study. The animals were divided into three experimental groups: 1. control group (wild-type zebrafish injected with control morpholino); 2. Itbp1 knock-down group (wild-type zebrafish injected with Itbp1 morpholino); 3. Rescue group (wild-type zebrafish co-injected with Itbp 1 morpholino and synthesized Itbp 1 mRNA). Each group was evaluated at two experimental periods: 3 DPF and 5 DPF stages.

\section{Microinjection of morpholino antisense oligo and mRNA}

Morpholino antisense oligos (MO) were designed to block translation and synthesis by Gene Tools, Inc. The Itbp1-MO was targeted to the 5'UTR of the Itbp1 mRNA, and the Itbp1-MO was bound to the 5'UTR (including ATG) of the Itbp1 mRNA. A standard control-MO was used as negative control (Table 1) .${ }^{28}$ Full-length cDNA was used and amplified to test knockdown specificity of the MO.26,28 Mutations in Itbp1-mRNA were introduced within the first 12 bases of open reading frame (ORF) (ATGCTgGTcTGt) without changing the sequences of amino acid so that mutated Itbp1 mRNA could mimic Itbp1 mRNA and it would not anneal to Itbp1-MO. The MOs and synthesized mRNA were injected into the yolk of embryos at the 1 -cell stage. Primers used are described in Table 2. Zebrafish embryos at the 1-cell stage were injected with $1 \mathrm{~nL}$ of Itbp1-MO at a concentration ranging from

Table 1- Morpholino antisense oligos

\begin{tabular}{cc}
\hline Morpholino & Sequence \\
\hline Itbp1-MO & 5'-AAACAATGATGTCCCACACGAGCAT-3 \\
control-MO & 5'-CCTCTTACCTCAGTTACAATTTATA- 3 \\
\hline
\end{tabular}

Table 2- Primers for the plasmid clone

\begin{tabular}{ccc}
\hline Construct & Primer & Sequence 5' -3' $^{\prime}$ \\
\hline Itbp1-mRNA & Itbp1-mm-ATG-EcoRI & GGAATTCcaccATGCTGGTCTGTGACATCATTGTT \\
& Itbp1-R-Xhol & CCGCTCGAGTCACTCTGTGCCCGTACTGGT \\
\hline
\end{tabular}

Table 3- Primers for the probes

\begin{tabular}{ccc}
\hline probe & Primer & Sequence 5'-3' $^{\prime}$ \\
\hline Itbp1 & PF-ltbp1-11 & ATGCCAGCTCTTTGGAAGTGA \\
sox9a & PR-Itbp1-743-T7 & taatacgactcactatagggGTTGATATCCACGCAGGCCA \\
& PF-sox9a-167 & CTCCTCGACCCCTACCTGAA \\
& PR-sox9a-761-T7 & taatacgactcactatagggAGATGTGGGTCTGTTCGCTG \\
& PF-ckma-404 & GTCACGGTGGATACAAGGCA \\
\hline
\end{tabular}

0.1 to $1 \mathrm{mM}$ and a control-MO was used as a negative control. The final dosages of Itbp1-MO are $0.25 \mathrm{pmol}$, $0.5 \mathrm{pmol}$, and $0.75 \mathrm{pmol}$. Zebrafish embryos at the 1 -cell stage were co-injected with Itbp1-mRNA(100 $\mathrm{pg} / \mathrm{embryo})$ and $/$ tbp 1 -MO(0.75 pmol/embryo) for the rescue experiments.

\section{Whole mount in situ hybridization}

Probes for Itbp1, sox9a, and ckma were cloned by polymerase chain reaction with primers presented in Table 3, whole mount in situ hybridization (WISH) was performed as described. ${ }^{29}$ The stained embryos were photographed with a Nikon SMZ1500 stereomicroscope.

\section{Alcian blue staining}

Embryos were fixed at 5 DPF, and cartilages were visualized by staining with alcian blue (SigmaAldrich; as described by Kimmel, et al. ${ }^{30}$ (1998) without using the $1.67 \%$ trypsin to digest the tissue.

\section{Statistical analysis}

Statistical analysis was performed using GraphPad Prism 5 (GraphPad Software Inc., San Diego, CA, USA). At 5 DPF, embryos were categorized according to zebrafish jaw malformation: normal, embryos with normal cartilages compared to uninjected embryos; mild, all cartilages are generally smaller and Meckel's cartilage is mildly malformed as well as positioned ventrally, leading to mandibular jaws opened along with the defects of ceratobranchial; moderate, deformities of Meckel's cartilage are severe, jaws are widely opened and the ceratobranchial is almost completely absent; severe, increased severity of the Meckel's cartilage distortion and the ceratobranchial is completely absent; malformed, embryos with malformed general 
morphology. The malformed category was excluded when the percentage of cartilage was estimated. The percentage of cartilage malformation was estimated by considering the total number of mild, moderate, and severe category, and then dividing it by the total number of normal, mild, moderate, and severe category. The mean grey value of WISH staining on the mandibular cartilage (outlined by red dot line) and mandibular surrounding muscle (outlined by red dot line) was measured via Imagej software. Measurements of lower jaw regions were conducted, including open mouth distance (Figure 3B), mandible length (Figure 3D), and mandible width (Figure 3F), the red double arrow represents the corresponding length. Each specific length was obtained by comparing the length of red double arrow with a scale bar that corresponds with the magnification of the alcian blue images.

Data are expressed as mean \pm standard deviation (SD). Comparison of different groups was performed by unpaired t-test to estimate data measurements. $P$-value less than $0.05(P<0.05)$ was considered statistically significant.

\section{Results}

\section{Itbp1 expresses at mandibular arch skeleton}

Since previous studies indicated that $\angle T B P 1$ is a crucial gene in the early embryo development and it is widely expressed in several systems, we suspected that $\angle T B P 1$ was expressed in craniofacial tissues. However, the expression pattern of $\angle T B P 1$ in craniofacial tissues remains unclear. Therefore, we decided to explore the expression pattern of $L T B P 1$ zebrafish ortholog in craniofacial tissues.

According to WISH of Itbp1 on zebrafish embryos, maternal transcripts were detected during the early developmental stage from 1 cell (Figure $1 \mathrm{~A}$ ) to shield (Figure 1C). At pharyngula period (1 DPF), Itbp 1 expressed at pharyngeal arches, brain, the most part of trunk, and the origin of internal organs (Figure 1D). In the developmental stage of protruding mouth (3 DPF), Itbp 1 was mainly detected in the mandibular jaw region and brain, but reduced expression also existed in the trunk and internal organs such as pancreas and gallbladder (Figure 1E, F). We performed 2-color WISH of Itbp1 with cartilage marker sox9a and muscle marker ckma, respectively, to further explore the expression pattern of $I t b p 1$ in the mandibular jaw region. sox9a is a marker of cranial neural crest that differs from cartilage, and sox9a participates in both determination of crest-derived chondrogenic lineages and morphogenesis of cartilage (2-3 DPF) ${ }^{31}$. ckma is a terminal differentiation marker for skeletal muscle, ${ }^{32}$ and $c k m a$ was expressed in muscles of the trunk, pectoral fin, head, and heart. ${ }^{33}$ According to the observation of the tissue section of zebrafish jaw, Itbp1 colocalized with both sox9a (Figure 1G-N) and $c k m a$ (Figure $1 \mathrm{~J}-\mathrm{L}$ ). The results indicated that Itbp1 was expressed in craniofacial tissues, and it was mainly concentrated in the zebrafish jaw cartilages and its surrounding muscles.

\section{Loss of Itbp1 function Results in Severe Jaw Malformation}

Based on the fact that Itbp 1 is expressed in the zebrafish mandibular jaw region, we speculated that Itbp 1 play a role in the formation of mandibular jaw. The loss of Itbp 1 function was mediated by morpholino knockdown to assess the function of Itbp1 during craniofacial development and mandibular jaw formation. We designed translation-blocking morpholino antisense oligonucleotides (MO) for Itbpl targeting regions in the $5^{\prime}$-UTR of the mRNA. MO with concentration ranging between 0.1 and $1 \mathrm{mM}$ was injected $1 \mathrm{~nL}$ into zebrafish embryos in 1-cell stage, and a control-MO was used as a negative control. Embryos injected with control-MO were indistinguishable from uninjected embryos. Embryos injected with Itbp1-MO exhibited several defects, including severe jaw malformation, pericardial edema, generally shortened body length and microphthalmia. At $5 \mathrm{DPF}$, Itbp 1 morphants presented significantly underdeveloped jaw regions and the mandibular jaws were widely opened (Figure 2E-F, I-J and M-N). We performed alcian blue staining to visualize the jaw cartilage skeleton. The staining revealed severe deformity of Meckel's cartilage along with the absence of ceratobranchial (Figure 2C-D, G-H, K-L and O-P). Further measurements of lower jaw regions were conducted, including open mouth distance (Figure 3B), mandible length (Figure 3D) and mandible width (Figure 3F), in order to assess the jaw defects. The measurement of this three dimensions showed that Itbp1 morphants decreased in both mandible length and width and it increased in open mouth distance compared to the control-MO group (Figure $3 \mathrm{~A}, \mathrm{C}, \mathrm{E}$ ). 
Interestingly, as Itbp1 MO dosages increase, both penetrance and severity of the jaw defects increases (Figure 2Q).

Rescue experiments with full-length Itbp 1 mRNA were carried out to confirm the specificity of Itbpl knockdown phenotype. Co-injection of nonhomologous Itbp1 mRNA with Itbp1 MO significantly rescued the jaw defects (Figure 4A-D). The rescued Itbp1-morphants presented increased jaw length and width, whereas their open mouth distance (Figure 4E-H) decreased, and also decreased the rescue of ceratobranchial
(Figure 4C). As most phenotypes were rescued when the mRNA encoding for Itbp 1 were co-injected in the Itbp $1 \mathrm{MO}$, the off-targeting effect of Itbp $1 \mathrm{MO}$ was ruled out, indicating the specificity of Itbp 1 knockdown phenotype.

\section{Loss of Itbp 1 function Lead to Abnormal} Cartilage and Muscle during Jaw Development

Since Itbp 1 is expressed in both jaw cartilages and jaw surrounding muscles, and the loss of Itbp1 function affected jaw development, we examined the two tissue
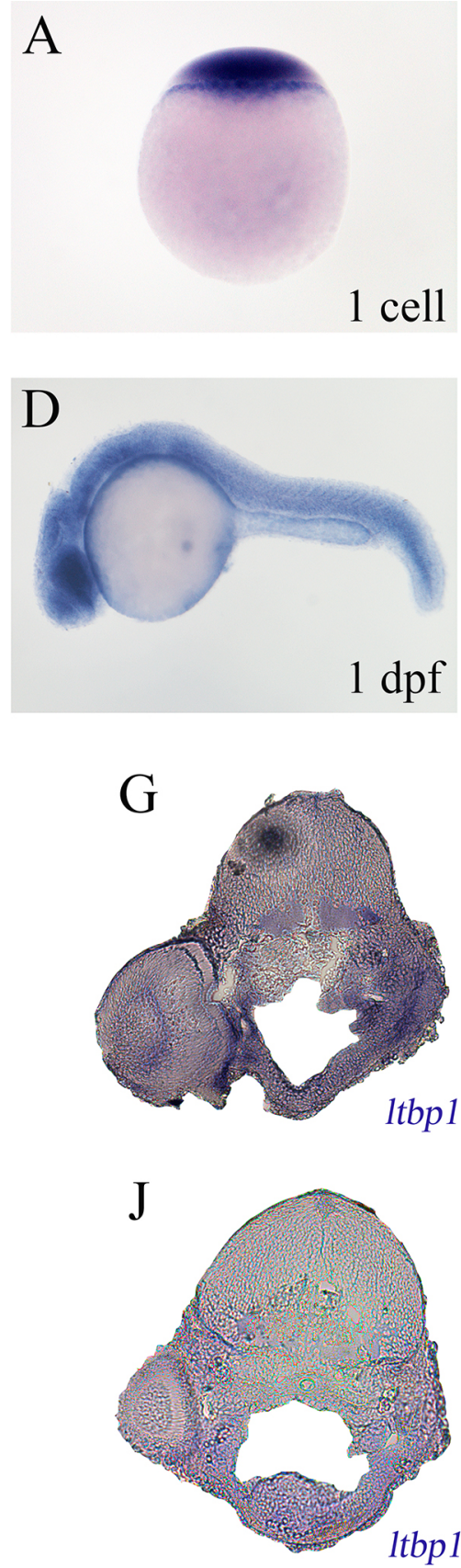
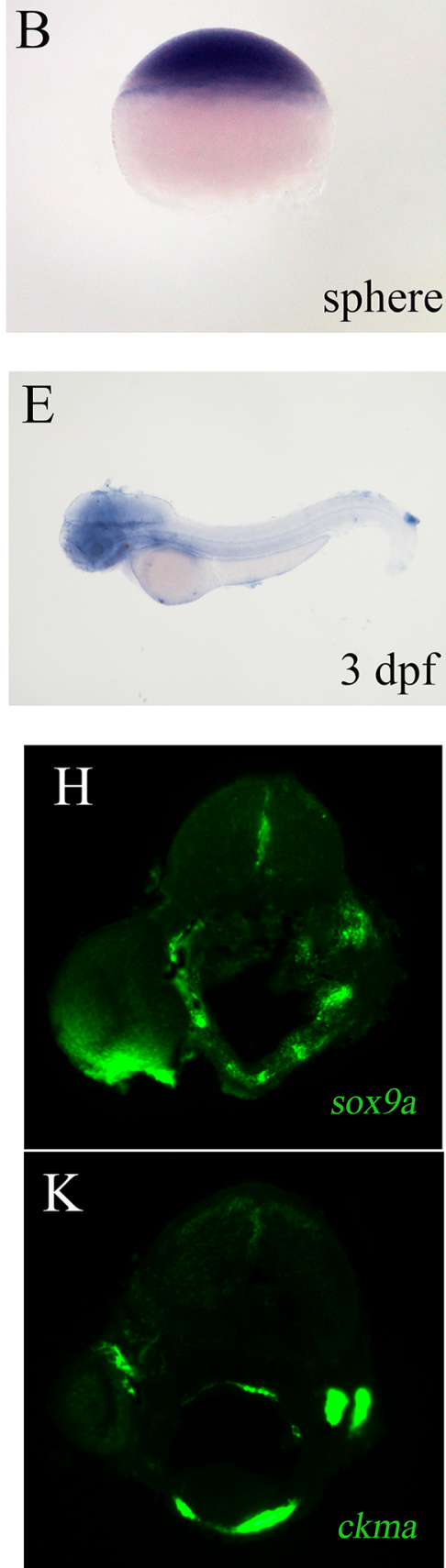
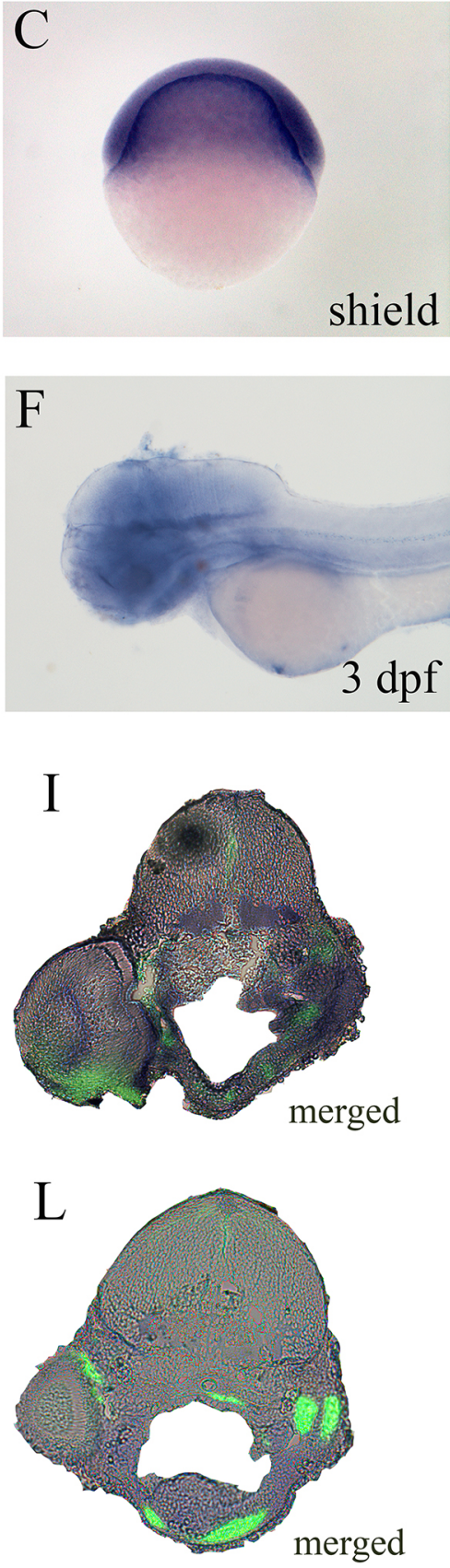

(A-F) Expression patterns analyzed by WISH with Itbp1 probes in the stages indicated (developmental stage: 1 cell , sphere, shield, 1 DPF and 3 DPF). Views are lateral. (G-L) Double-dye WISH of Itbp1 with either sox9a or ckma fluorescence-probe. Itbp1 colocalized with both sox9a (G-I) and ckma (J-L) in the 3-DPF embryo transections.

Figure 1- Expression patterns of Itbp1 
in Itbp1-morphants during jaw formation. Because 3 DPF is the stage of protruding-mouth stage, when the developing cartilages of the pharyngeal skeleton such as Meckel's cartilage, has grown dramatically, we chose 3 DPF as developmental stage to examine the effects of Itbp1 knockdown. We conducted WISH of sox9a and ckma respectively on control-MO embryos, Itbp1-morphants, as well as Itbp1 rescued morphants at 3 DPF. The expression of sox9a was detected in mesenchymal condensation of pharyngeal arches

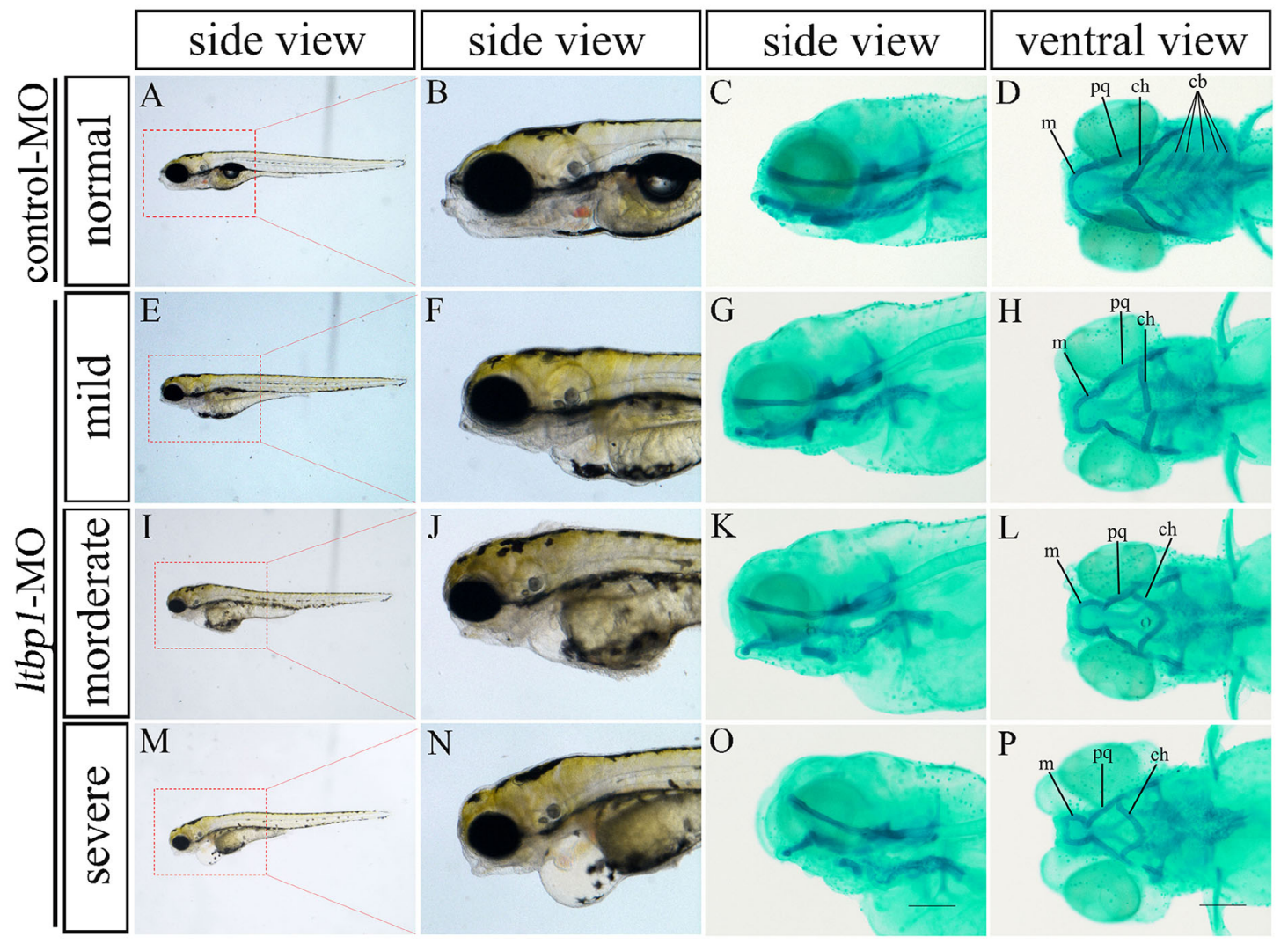

Q

ltbpl-MO

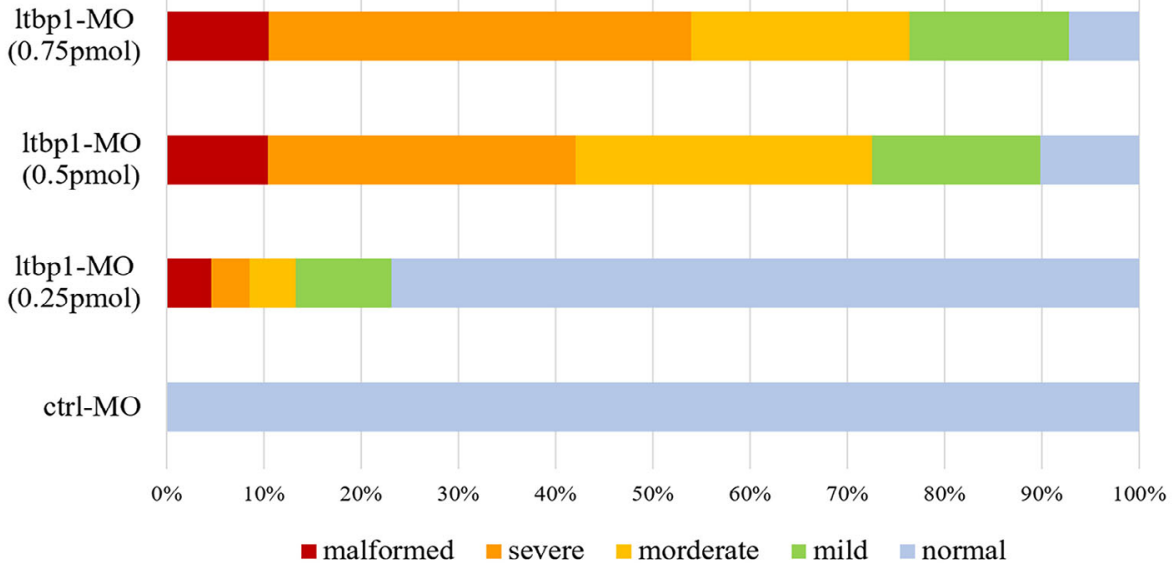

Injection of Itbp1-MO caused marked jaw defects compared to standard control embryos at 5 DPF (A,E,I ,M and B,F,J,N). Larvae at 5 DPF were stained with alcian blue to observe craniofacial cartilage structures $(C, G, K, O$ and $D, H, L, P)$. Views are lateral and ventral. ( $Q)$ Quantification of craniofacial phenotypes of Itbp1 morphant. We defined five categories regarding jaw malformation of zebrafish: (A-D) normal, embryos with normal cartilages compared to uninjected embryos; $(\mathrm{E}-\mathrm{H})$ mild, all cartilages are generally smaller, and Meckel's cartilage is mildly malformed as well as positioned ventrally, leading to opened mandibular jaws as well as defects of ceratobranchial; (I-L) moderate, deformities of Meckel's cartilage are more severe and jaws are widely opened, and the ceratobranchial are almost completely absent; (M-P) severe, severe distortion of Meckel's cartilage and the ceratobranchial are completely absent; malformed, embryos with malformed general morphology. ch, ceratohyal; m, Meckel's cartilage; pq, palatoquadrate; cb, ceratobranchial. Scale bars: 200 $\mu m$ (C-D, G-H, K-L and O-P).

Figure 2- Depletion of Itbp1 causes severe jaw malformation in zebrafish 
and jaw cartilages in control and rescued morphants, while significantly decreased in Itbp 1-morphants (Figure 5A-C and G). Similarly, expression of ckma was shown in mandibular region of craniofacial muscle in control and rescued morphants, but severely reduced in Itbp1-morphants (Figure 5D-F and $\mathrm{H}$ ). The WISH of sox9a and ckma indicated that Itbp1 function loss affected jaw development in both jaw cartilage and
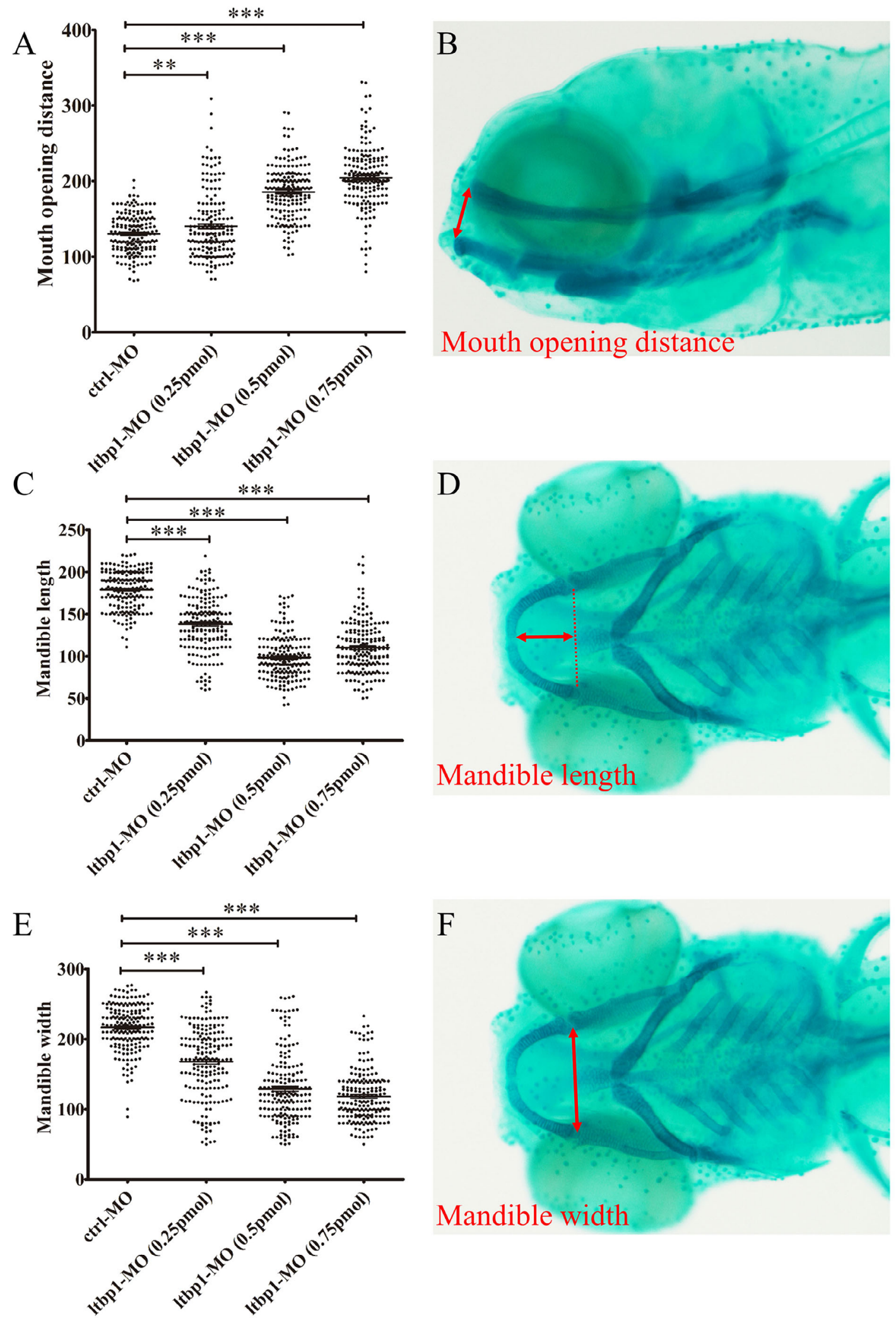

Figure 3- Three dimensions (3D) measurements of mandibular jaw malformation

Red double arrow represents the (B) mouth-opening distance, (D) mandible length, and (F) mandible width. Scatter plots showed an increase of mouth-opening distance and a decrease of mandible length and width in ( $A, C, E)$ Itbp1-morphant. $N=3$, $n=60$ per group, ${ }^{* *} \mathrm{P}<0.01,{ }^{* * *} \mathrm{P}<0.001$. 

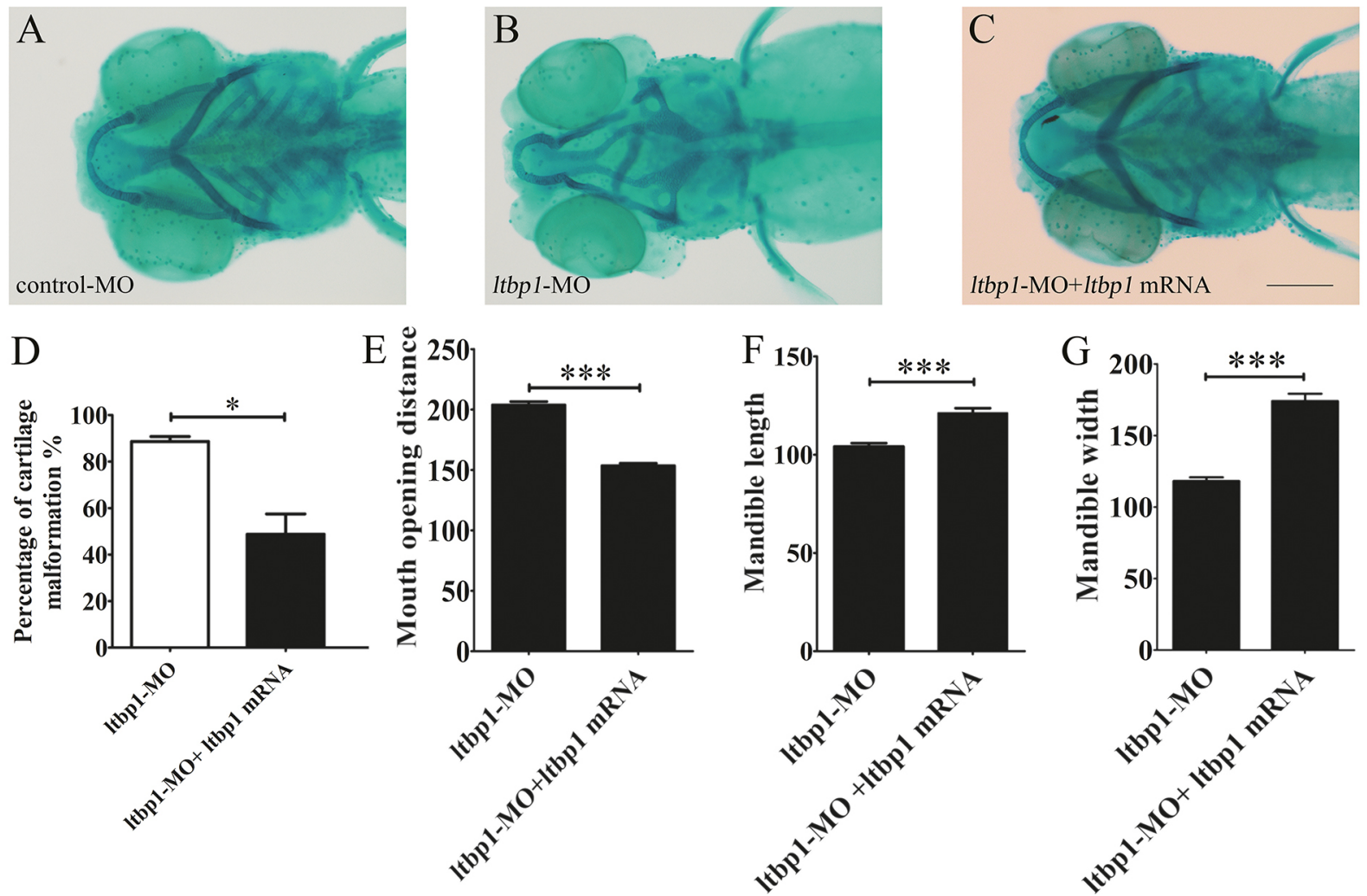

RNA rescue experiments for Itbp1 knockdown in zebrafish using Itbp1 mRNA. (A-C) Embryos were stained with alcian blue at 5 DPF to visualize craniofacial cartilage structures. All views are ventral. Jaw malformation induced by Itbp 1 knockdown was significantly rescued by the co-injection of Itbp1 mRNA (D), including reduction in mouth opening distance $(F)$, recover in mandible length $(G)$ and width $(H)$. (E-G) Data represent the mean $\pm S D, N=3, n=60$ per group, $\left({ }^{*} P<0.05,{ }^{* * *} \mathrm{P}<0.001\right)$. Error bars indicate standard error. Scale bars: $200 \mu \mathrm{m}$ (A-C). MO, morpholino antisense oligo.

Figure 4- Specificity of Itbp1 knockdown phenotype

muscle aspects.

\section{Discussion}

$\angle T B P 1$ is an important extracellular matrix protein that regulates the bioactivity of TGF- $\beta$ and also exists as microfibrillar structure of bone and connective tissue. According to recent researches, the expression of Itbp 1 is widely distributed in different tissues. ${ }^{2,3}$ Gene disruption experiments have shown that $\angle T B P 1$ play roles in several fields, including the development and formation of cardiovascular system, craniofacial system, reproduction system as well as functions in both bone and connective tissue. ${ }^{21-24}$ In this study, we explored the expression pattern of Itbp 1 using zebrafish. The results of Itbp1 WISH showed detectable maternal transcripts from 1 cell to shield stage, which indicated that Itbp 1 is necessary during the early embryo development. In the pharyngula period (1 DPF), Itbp1 was extensively expressed in the pharyngeal arches, brain, most part of trunk and the origin of some internal organs. In the developmental stage of protruding mouth ( $3 \mathrm{DPF}$ ), Itbp 1 expression was mainly in the mandibular jaw region and brain, while reduced expression was also present in the trunk and internal organs such as pancreas and gallbladder. The Itbp 1 WISH results are according with previously reported widespread tissue expression. Since Itbp 1 is strongly expressed in the mandibular jaw region at 3 DPF, and the zebrafish jaw region mainly consists of cartilages and muscles. We carried out 2-colour WISH of Itbp 1 with cartilage marker sox9a and muscle marker ckma in order to specify the target tissue of Itbp 1 expression. The results showed that Itbp 1 colocalized with both sox9a and ckma, indicating that Itbp 1 concentrates in the zebrafish jaw cartilages and its surrounding muscles.

Considering that the function of a gene is usually associated with its expression pattern, we hypothesize Itbp 1 may have its function in the craniofacial development of zebrafish. According to previous studies, different strategies of Ltbp 1 knockout mice presented several phenotypes, including heart defects, craniofacial deformities, and impaired reproduction. ${ }^{21-24}$ However, the discrepancy of the variable phenotypes 

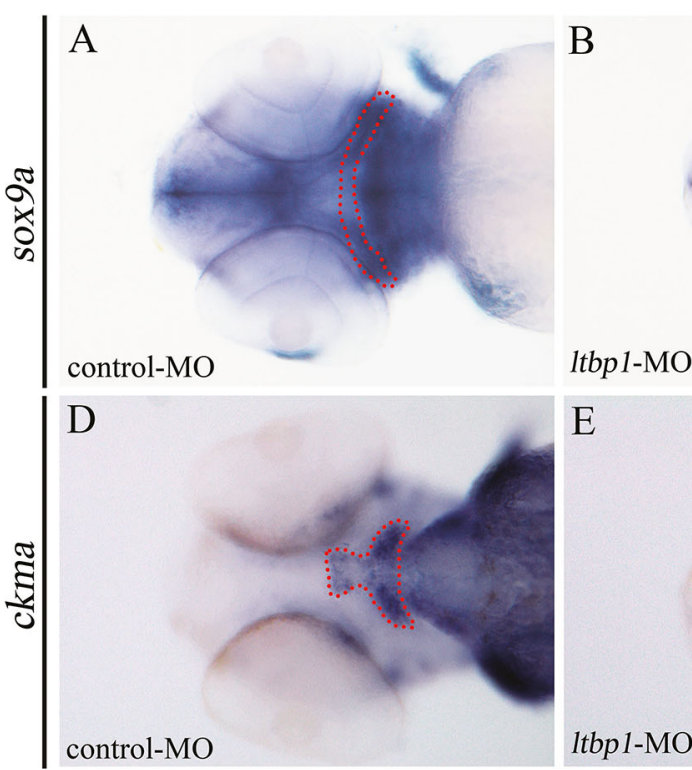

$\mathrm{E}$

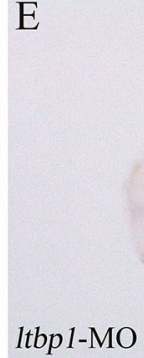

$\mathrm{C}$

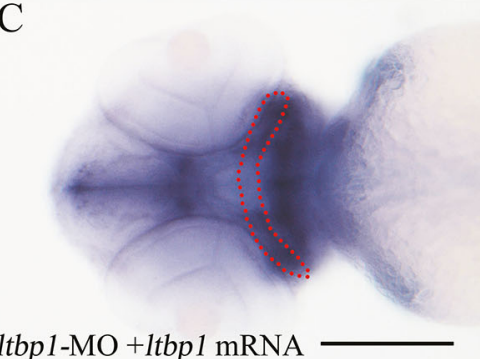

1tbp1-MO +ltbp1 mRNA

$\mathrm{F}$

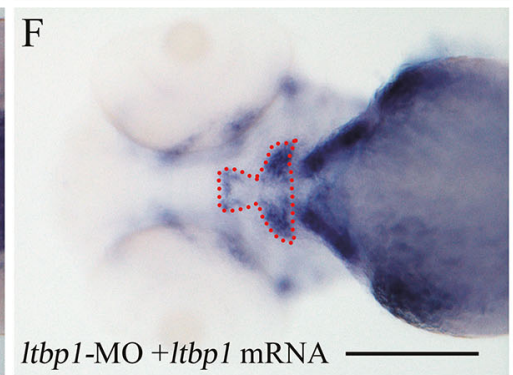

G

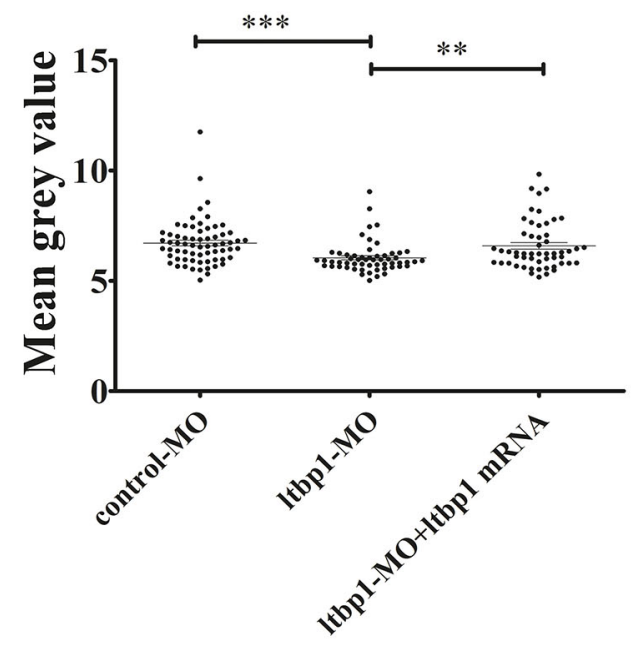

$\mathrm{H}$

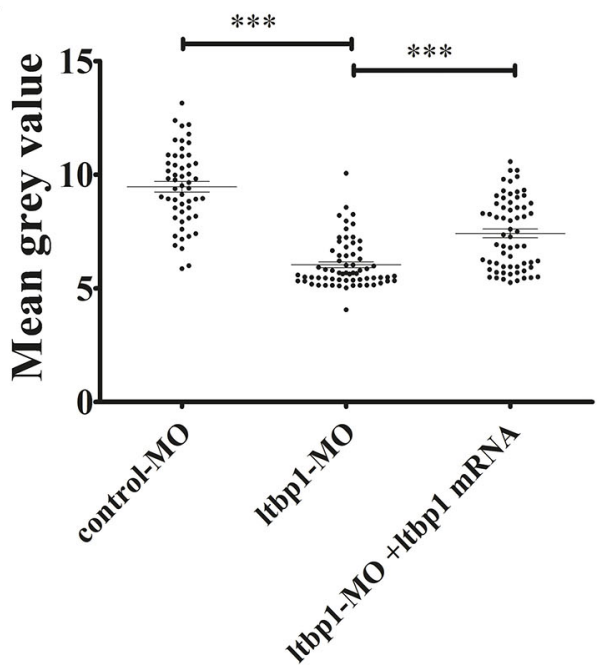

Bright-field views of in situ hybridization of cartilage marker sox9a and muscle marker ckma expression in zebrafish larvae at 72 hour postfertilization (hpf). sox9a expression in (A) control-MO, (B) Itbp1-MO, (C) Itbp1-MO with Itbp1 mRNA from ventral view. sox9a expression was reduced in mandibular cartilage (outlined by red dot line) of 72-hpf (B) Itbp1-morphant. The reduction of sox9a expression in morphants was rescued by co-injection of (C) Itbp1 RNA. (G) sox9a expression showed significant differences among morphants and control embryos. ckma expression in (D) control-MO, (E) Itbp1-MO, (F) Itbp1-MO with Itbp1 mRNA from ventral view. ckma expression was reduced in mandibular surrounding muscle (outlined by red dot line) of 72-hpf (E) Itbp1-morphant. The reduction of ckma expression in morphants was rescued by co-injection of (C) Itbp1 RNA. (H)ckma expression showed significant differences among morphants and control embryos. $(G, H) N=3, n=15$ per group, $\left({ }^{* *} P<0.01^{* * *} P<0.001\right)$. Scale bar: $200 \mu \mathrm{m}(A-F)$. MO, morpholino antisense oligo.

Figure 5- Loss of Itbp1 function affects formation of both craniofacial cartilage and muscle

in $L t b p 1$ gene-manipulated mice is complicated and ambiguous. To repeat the phenotype in another model, we performed morpholino based knockdown of Itbp1 using zebrafish.

Generally, Itbp1 morphants presented shortened body length, heart edema, and severe jaw malformation. At $5 \mathrm{DPF}$, Itbp 1 morphants manifested significantly underdeveloped jaw regions and the mandibular jaw was widely opened. These phenotypes are consistent with the observation of Ltbp1 knockout mice which were built by different strategies.

In this study, we focused on jaw defects of Itbp 1 morphants, since this craniofacial aspect is the most significant observed in this phenotype, remaining unclear. Alcian blue staining of 5 DPF Itbp 1 morphants revealed severe deformity of Meckel's cartilage as well as the absence of ceratobranchial. We conducted three dimensional measurements of lower jaw regions to assess the jaw defects, and the Itbp 1 morphants decreased both mandible length and width and their open mouth distance increased. Similar phenotype was previously observed in the Ltbp1-null mice reported by Drews, et al.23 (2008), which showed modified facial profile with shortened snouts and 
shortened maxilla and mandible. We also found that both penetrance and severity of Itbp1-morphants defects of jaw increases as the dosages of Itbp $1 \mathrm{MO}$ increases. Meanwhile, most phenotypes were rescued by co-injection of nonhomologous Itbp 1 mRNA with Itbp $1 \mathrm{MO}$, therefore the off-targeting effect of Itbp 1 MO was ruled out, indicating the specificity of Itbp 1 morphant phenotype. Since Itbp 1 is expressed in both jaw cartilages and surrounding muscles, we speculated that the jaw deformities could derive from the defects of either or both the two tissues. In order to address possible major aspect, we checked both cartilage and muscle markers in Itbp1-morphants. Both expression of sox9a and ckma was significantly decreased in Itbp1-morphants compared to control-MO and Itbp1 rescued morphants, indicating that knockdown of Itbp1 affected jaw development in both jaw cartilage and muscle aspects.

Considering that some previous studies have found that latent TGF- $\beta$ is present in the matrix of chondrocytes and that $\angle T B P 1$ is responsible for storing TGF- $\beta$ complex in the matrix. ${ }^{6,7}$ TGF- $\beta 1$ and their receptors have been found to be related to zebrafish craniofacial bone and cartilage development. ${ }^{34}$ The cartilage development may be affected by indirect regulating function of $\angle T B P 1$ and TGF- $\beta 1$. Besides, $\angle T B P 1$ may directly affect chondrocytes as an important extracellular matrix protein. Furthermore, $\angle T B P 1$ may participate in the muscle formation as microfibrillar structures of connective tissue ${ }^{8}$. Future studies will investigate the biological activity of TGF- $\beta$ and extracellular fibrillar structure whether they are compromised or not. Although the specific mechanisms require further research, our results have extended the understanding of the important role of $\angle T B P 1$ especially in craniofacial development, easing future investigations on the mechanisms of $L T B P 1$ gene function.

\section{Conclusion}

Our experiments found that Itbp 1 is expressed in zebrafish mandibular jaw cartilages and the surrounding muscles in addition to the previously reported tissues. Also, the Itbp1 knockdown zebrafish presented phenotypes consistent with Ltbp1 knockout mice. Lastly, loss of Itbp1 function lead to significant mandibular jaw defects and affect both jaw cartilages and surrounding muscles.

\section{Conflict of interest}

Nothing to declare.

\section{Funding}

Funding for this project is provided by the National Natural Science Foundation of China $(81371129,81670973,81970921)$ and Shanghai Sailing Program (20YF1453500).

\section{Acknowledgements}

We are grateful to Miaomiao Jin, Fang Yuan, and Weilai Huang for helpful discussion and Ruikun $\mathrm{Hu}$, Guanliang Huang for fish maintenance. And we are extremely grateful to Professor Cao Ying for constructive advice for the design of the experiment and the modification of the paper. The authors declare no potential conflicts of interest regarding the authorship and/or publication of this article.

\section{Authors' contributions}

Xiong, Yiting: Conceptualization (Equal); Data curation (Equal); Formal analysis (Equal); Funding acquisition (Equal); Investigation (Equal); Methodology (Equal); Project administration (Equal); Resources (Equal); Software (Equal); Supervision (Equal); Validation (Equal); Visualization (Equal); Writing-original draft (Equal); Writing-review \& editing (Equal). Sun, Rongrong: Conceptualization (Equal); Methodology (Equal); Project administration (Equal); Supervision (Equal); Writing-original draft (Equal); Writing-review \& editing (Equal). LI, Jingyu: Data curation; (Equal); Formal analysis (Equal). Wu, Yue: Data curation (Equal); Formal analysis (Equal). Zhang, Jingju: Conceptualization (Equal); Methodology (Equal); Project administration (Equal); Supervision (Equal); Writing-original draft (Equal); Writing-review \& editing (Equal)

\section{Reference}

1- Koski C, Saharinen J, Keski-Oja J. Independent promoters regulate the expression of two amino terminally distinct forms of latent transforming growth factor-beta binding protein-1 (LTBP-1) in a cell type-specific manner. J Biol Chem. 1999;274(46):32619-30. doi:10.1074/jbc.274.46.32619. 
2- Weiskirchen R, Moser M, Günther K, Weiskirchen S, Gressner AM. The murine latent transforming growth factor-beta binding protein $(L t b p-1)$ is alternatively spliced, and maps to a region syntenic to human chromosome 2p21-22. Gene. 2003;308:43-52. doi:10.1016/ s0378-1119(03)00464-5

3- Faraoni EY, Camilletti MA, Abeledo-Machado A, Laura DR, Ratner F, De Fino $F$, et al. Sex differences in the development of prolactinoma in mice overexpressing hCG $\beta$ : role of TGF $\beta 1$. J Endocrinol. 2017;232(3):53546. doi:10.1530/JOE-16-0371

4- Akhurst RJ, Lehnert SA, Faissner A, Duffie E. TGF beta in murine morphogenetic processes: the early embryo and cardiogenesis. Development. 1990;108(4):645-56.

5- Nakajima Y, Miyazono K, Nakamura H. Immunolocalization of latent transforming growth factor-beta binding protein-1 (LTBP1) during mouse development: possible roles in epithelial and mesenchymal cytodifferentiation. Cell Tissue Res. 1999;295(2):257-67. doi:10.1007/ s004410051232

6- Pedrozo HA, Schwartz Z, Gomez R, Ornoy A, Xin-Sheng W, Dallas $\mathrm{SL}$, et al. Growth plate chondrocytes store latent transforming growth factor (TGF)-beta 1 in their matrix through latent TGF-beta 1 binding protein-1. J Cell Physiol. 1998;177(2):343-54. doi:10.1002/ (SICI)1097-4652(199811)177:2<343: :AID-JCP16>3.0.CO;2-A 7- Pedrozo HA, Schwartz Z, Mokeyev T, Ornoy A, Xin-Sheng W, Bonewald $L F$, et al. Vitamin D3 metabolites regulate LTBP1 and latent TGF-beta1 expression and latent TGF-beta1 incorporation in the extracellular matrix of chondrocytes. J Cell Biochem. 1999;72(1):151-65.

8- Dallas SL, Keene DR, Bruder SP, Saharinen J, Sakai LY, Mundy GR, et al. Role of the latent transforming growth factor beta binding protein 1 in fibrillin-containing microfibrils in bone cells in vitro and in vivo. J Bone Miner Res. 2000;15(1):68-81. doi:10.1359/jbmr.2000.15.1.68 9- Ota T, Fujii M, Sugizaki T, Ishii M, Miyazawa $K$, Aburatani $H$, et al. Targets of transcriptional regulation by two distinct type I receptors for transforming growth factor-beta in human umbilical vein endothelial cells. J Cell Physiol. 2002;193(3):299-318. doi:10.1002/jcp.10170 10- Vittal R, Mickler EA, Fisher AJ, Zhang C, Rothhaar K, Gu H, et al. Type $V$ collagen induced tolerance suppresses collagen deposition, TGF- $\beta$ and associated transcripts in pulmonary fibrosis [published correction appears in PLoS One. 2018;13(12):e0209107]. PLoS One. 2013;8(10):e76451. Published 2013 Oct 21. doi:10.1371/journal. pone.0076451

11- Nakajima Y, Miyazono K, Kato M, Takase M, Yamagishi T, Nakamura H. Extracellular fibrillar structure of latent TGF beta binding protein-1: role in TGF beta-dependent endothelial-mesenchymal transformation during endocardial cushion tissue formation in mouse embryonic heart. J Cell Biol. 1997;136(1):193-204. doi:10.1083/jcb.136.1.193

12- Nakano M, Arai E, Nakajima Y, Nakamura H, Miyazono K, Hirose T. Immunohistochemical study of chondrolipoma: possible importance of transforming growth factor (TGF)-betas, latent TGFbeta binding protein-1 ( $L T B P-1)$, and bone morphogenetic protein (BMP) for chondrogenesis in lipoma. J Dermatol. 2003;30(3):189-95. doi:10.1111/j.1346-8138.2003.tb00370.x

13- Gui Y, Murphy LJ. Interaction of insulin-like growth factor binding protein-3 with latent transforming growth factor-beta binding protein-1. Mol Cell Biochem. 2003;250(1-2):189-95. doi:10.1023/a:1024990409102

14- Ge G, Greenspan DS. BMP1 controls TGF beta1 activation via cleavage of latent TGFbeta-binding protein. J Cell Biol. 2006;175(1):111-20. doi:10.1083/jcb.200606058

15- Maurya VK, Jha RK, Kumar V, Joshi A, Chadchan S, Mohane JJ, et al. Transforming growth factor-beta 1 (TGF-B1) liberation from its latent complex during embryo implantation and its regulation by estradiol in mouse [published correction appears in Biol Reprod. 2014;91(6):147]. Biol Reprod. 2013;89(4):84. Published 2013 Oct 10. doi:10.1095/ biolreprod.112.106542
16- Zhao Q, Zheng K, Ma C, Li J, Zhuo L, Huang W, et al. PTPS facilitates compartmentalized $\angle T B P 1 \mathrm{~S}$-nitrosylation and promotes tumor growth under hypoxia. Mol Cell. 2020;77(1):95-107.e5. doi:10.1016/j. molcel.2019.09.018

17- Robertson IB, Handford PA, Redfield C. Backbone ${ }^{1 \mathrm{H}},{ }^{13} \mathrm{C}$ and ${ }^{15} \mathrm{~N}$ resonance assignment of the C-terminal EGF-cbEGF pair of $\angle T B P 1$ and flanking residues. Biomol NMR Assign. 2014;8(1):159-63. doi:10.1007/ s12104-013-9474-6.

18- Robertson IB, Handford PA, Redfield C. NMR spectroscopic and bioinformatic analyses of the $\angle T B P 1 \mathrm{C}$-terminus reveal a highly dynamic domain organisation. PLoS One. 2014;9(1):e87125. doi:10.1371/ journal.pone.0087125

19- Chen H, Cai W, Chu ES, Tang J, Wong CC, Wong SH, et al. Hepatic cyclooxygenase-2 overexpression induced spontaneous hepatocellular carcinoma formation in mice. Oncogene. 2017;36(31):4415-26. doi: 10.1038 /onc. 2017.73

20- Hubmacher D, Wang LW, Mecham RP, Reinhardt DP, Apte SS. Adamts 12 deletion results in bronchial fibrillin microfibril accumulation and bronchial epithelial dysplasia--a novel mouse model providing insights into geleophysic dysplasia. Dis Model Mech. 2015;8(5):48799. doi:10.1242/dmm.017046

21- Todorovic V, Frendewey D, Gutstein DE, Chen Y, Freyer L, Finnegan $\mathrm{E}$, et al. Long form of latent TGF-beta binding protein 1 (Ltbp1L) is essential for cardiac outflow tract septation and remodeling. Development. 2007;134(20):3723-32. doi:10.1242/dev.008599 22- Horiguchi M, Todorovic V, Hadjiolova K, Weiskirchen R, Rifkin DB. Abrogation of both short and long forms of latent transforming growth factor- $\beta$ binding protein- 1 causes defective cardiovascular development and is perinatally lethal. Matrix Biol. 2015;43:61-70. doi:10.1016/j. matbio.2015.03.006

23- Drews F, Knöbel S, Moser M, Muhlack KG, Mohren S, Stoll C, et al. Disruption of the latent transforming growth factor-beta binding protein-1 gene causes alteration in facial structure and influences TGF-beta bioavailability. Biochim Biophys Acta. 2008;1783(1):34-48. doi:10.1016/j.bbamcr.2007.08.004

24- Dietzel E, Weiskirchen S, Floehr J, Horiguchi M, Todorovic V, Rifkin DB, et al. Latent TGF- $\beta$ binding protein-1 deficiency decreases female fertility. Biochem Biophys Res Commun. 2017;482(4):1387-92. doi: $10.1016 /$ j.bbrc.2016.12.046

25- Mork L, Crump G. Zebrafish craniofacial development: a window into early patterning. Curr Top Dev Biol. 2015;115:235-269. doi:10.1016/ bs.ctdb.2015.07.001

26- Machado RG, Eames BF. Using zebrafish to test the genetic basis of human craniofacial diseases. J Dent Res. 2017;96(11):1192-9. doi: $10.1177 / 0022034517722776$

27- Kimmel CB, Ballard WW, Kimmel SR, Ullmann B, Schilling TF. Stages of embryonic development of the zebrafish. Dev Dyn. 1995;203(3):253-310. doi:10.1002/aja.1002030302

28- Stainier DY, Raz E, Lawson ND, Ekker SC, Burdine RD, Eisene JS, et al. Guidelines for morpholino use in zebrafish. PLoS Genet. 2017;13(10):e1007000. doi:10.1371/journal.pgen.1007000

29- Thisse $B$, Thisse $C$. In situ hybridization on whole-mount zebrafish embryos and young larvae. Methods Mol Biol. 2014;1211:53-67. doi:10.1007/978-1-4939-1459-3_5

30- Kimmel CB, Miller CT, Kruze G, Ullmann B, BreMiller RA, Larison KD, et al. The shaping of pharyngeal cartilages during early development of the zebrafish. Dev Biol. 1998;203(2):245-63. doi:10.1006/ dbio.1998.9016

31- Yan YL, Willoughby J, Liu D, Crump JG, Wilson C, Miller CT, et al. A pair of sox: distinct and overlapping functions of zebrafish sox 9 coorthologs in craniofacial and pectoral fin development. Development. 2005;132(5):1069-83. doi:10.1242/dev.01674 
32- Xu Y, He J, Wang X, Lim TM, Gong Z. Asynchronous activation of 10 muscle-specific protein (MSP) genes during zebrafish somitogenesis. Dev Dyn. 2000;219(2):201-15. doi:10.1002/10970177(2000)9999:9999<: :aid-dvdy1043>3.3.c0;2-9

33- Thisse B, Pflumio S, Fürthauer M, Loppin B, Heyer V, Degrave A, et al. Expression of the zebrafish genome during embryogenesis (NIH R01 RR15402) [Internet]. Eugene: ZFIN; 2001 [cited 2020 Aug 5]. Available from: https://zfin.org/ZDB-PUB-010810-1
34- Zhang Y, Ji D, Li L, Yang S, Zhang H, Duan X. ClC-7 Regulates the pattern and early development of craniofacial bone and tooth. Theranostics. 2019;9(5):1387-400. doi:10.7150/thno.29761 